\section{Review}

Correspondence

Verena Seidl-Seiboth

verena.seidl@tuwien.ac.at

\title{
Self versus non-self: fungal cell wall degradation in Trichoderma
}

\author{
Sabine Gruber and Verena Seidl-Seiboth
}

Research Area Gene Technology and Applied Biochemistry, Institute of Chemical Engineering, Vienna University of Technology, Gumpendorfer Strasse 1a, 1060 Vienna, Austria

\begin{abstract}
Lysis of the prey's cell wall is one of the key steps during mycoparasitism. Genome analysis of two mycoparasitic Trichoderma species, T. atroviride and T. virens, revealed an expanded arsenal of genes encoding enzymes potentially involved in cell wall hydrolysis. Glycoside hydrolase family 18 , which contains all fungal chitinases, is the largest family of carbohydrate-active enzymes in mycoparasitic Trichoderma species. However, in addition to their aggressive functions during mycoparasitism, the roles of chitinases and other cell wall degrading enzymes also include remodelling and recycling of the fungus's own cell wall. In this review we discuss current knowledge about fungal cell wall degrading enzymes in Trichoderma and how the fungus distinguishes between self- and non-self fungal cell wall degradation. In the past few years, the chitinolytic enzyme machinery of Trichoderma has been used as a model system to address this question. Gene expression profiles of most investigated chitinases indicate an overlap of functions of the respective enzymes and an involvement in both self- and non-self fungal cell wall degradation. Similar sets of enzymes appear to be involved in mycoparasitism, exogenous chitin decomposition and recycling of the fungus's own cell wall. Thus, we hypothesize that the regulation of self and non-self fungal cell wall degradation is not due to a speciation of individual chitinases. Rather, we hypothesize that it is regulated by substrate accessibility due to cell wall protection in healthy hyphae vs deprotection during mycoparasitic attack, hyphal ageing and autolysis.
\end{abstract}

\section{Introduction}

Mycoparasites are fungi that parasitize other fungi and can - by analogy to plant parasites - be grouped into two broad categories: biotrophic and necrotrophic mycoparasites. In biotrophic mycoparasitism the fungus can establish a specialized feeding relationship, usually by producing haustoria to penetrate and absorb nutrients from living fungal hyphae. In contrast, in necrotrophic mycoparasitism the fungus invades and destroys other fungal cells and feeds on the dead cell contents (Deacon, 2006). Several Trichoderma spp. have been long known for their potent necrotrophic mycoparasitic abilities (Benítez et al., 2004) and are therefore used as model systems to study the mechanisms of mycoparasitism. The different stages during the mycoparasitic attack include sensing of the prey fungus, attachment to the prey's hyphae, degradation of the prey's cell wall and ultimately killing of the prey. Cell wall degradation during mycoparasitism is mediated by a set of enzymes including chitinases, $\beta-(1,4)-, \beta-(1,3)$ - and $\beta$ $(1,6)$-glucanases, and proteases. Several members from each of these classes have been shown to be involved in

Abbreviations: CAZyme, carbohydrate-active enzyme; CBM, carbohydrate-binding module; $\mathrm{CE}$, carbohydrate esterase; $\mathrm{GH}$, glycoside hydrolase; GT, glycosyltransferase; PL, polysaccharide lyase. mycoparasitism and/or to be induced under mycoparasitism-related growth conditions (Benítez et al., 2004). Genome analysis enabled assessment of the total numbers of cell wall degrading enzymes encoded in the genomes of Trichoderma spp. and unravelled an even more complex enzymic degradation machinery for fungal cell walls than previously anticipated (Kubicek et al., 2011).

Trichoderma spp. can parasitize a wide range of fungi, including some species that are relatively closely related to Trichoderma in the fungal kingdom, e.g. Botrytis cinerea and some Fusarium spp. Cell wall lytic enzymes, however, are not only involved in mycoparasitism or chitin degradation for nutritional purposes, but are also important for cell wall recycling during ageing and autolysis as well as cell wall remodelling during active growth (e.g. hyphal branching). These aspects were somewhat disregarded in the past in mycoparasitism research. In view of the large numbers of cell wall lytic enzymes in the Trichoderma genomes we address the following question in this review: Can enzymes be differentiated that are either only used for the attack of other fungal cell walls or for remodelling and recycling of the fungus's own cell wall? Chitinases are the best-studied group of cell wall lytic enzymes in Trichoderma. In this review we use them as an example to emphasize the multiple roles of cell wall lytic enzymes. A 
survey of the chitinase literature including recent data on subgroup $\mathrm{C}$ chitinase gene regulation shows that there is evidence for many individual chitinases being involved in self and non-self cell wall degradation. Thus, it appears that factors related to substrate accessibility, e.g. protection of the fungal cell wall, are the determinants for self vs non-self cell wall degradation. A better understanding of the regulation of cell wall degrading enzymes will help to elucidate the events that trigger mycoparasitic attack. This will significantly expand our understanding of the events that initiate the mycoparasitic response in Trichoderma.

\section{Lessons about fungal cell wall degradation from Trichoderma genomes CAZomes}

The CAZome of an organism describes the total numbers and functional classification of carbohydrate-active enzymes (CAZymes) based on its genome annotation. The different categories of CAZymes, which are further grouped into different families, include glycoside hydrolases (GHs), glycosyltransferases (GTs), polysaccharide lyases (PLs), carbohydrate esterases (CEs) and carbohydrate-binding modules (CBMs) (http://www.cazy.org; Cantarel et al., 2009).

CAZome analysis of the recently sequenced genomes of the mycoparasites Trichoderma atroviride and Trichoderma virens revealed that these two fungi have high numbers of GHs in comparison to other filamentous fungi, whereas the total number of GHs is below average in the saprotrophic species Trichoderma reesei. T. atroviride and T. virens also have high numbers of CBMs and most of them are found in GH proteins, indicating a good ability of these enzymes to adhere to insoluble substrates (Kubicek et al., 2011). The numbers of GTs, PLs and CEs in the three sequenced Trichoderma spp. are similar to those in other filamentous fungi. Detailed CAZome comparison showed that the total numbers of GHs are higher in the mycoparasites $T$. atroviride and $T$. virens because of expansion of a few $\mathrm{GH}$ families. As can be seen in Table 1, most of these enzymes are associated with fungal cell wall degradation. Although chitinase and glucanase genes had already been cloned from Trichoderma spp., CAZome analysis revealed genes for many more members of the respective GH families. Further, several expanded CAZyme families were identified whose activities can also be related to fungal cell wall degradation but have not been studied in Trichoderma so far.

The structural scaffold of the fungal cell wall is composed of chitin - a $\beta$-(1,4)-linked $N$-acetylglucosamine polymer and $\beta$-(1,3)-glucan (Latgé, 2007). CAZome analysis showed that the numbers of chitinases and $\beta$ - $(1,3)$-glucanases are indeed higher in mycoparasitic Trichoderma spp. than in other filamentous fungi. Chitin and $\beta$-(1,3)-glucan together form the fibrillar, 'alkali-insoluble' fraction of fungal cell walls that is embedded in the amorphous, 'alkali-soluble' fraction, which consists of $\alpha$-glucans, galactomannans and other carbohydrate polymers. Unfortunately only a few details about the cell wall composition of
Trichoderma are available. In the 1970s the cell wall composition of spores and mycelia of Trichoderma viride (strain CECT 2423) was compared (Benítez et al., 1975, 1976). Melanin, which in other fungi is associated with chitin, was shown to replace this polymer in the spore wall of T. viride, and chitin was found only in mycelial cell walls. However, similar findings have also been reported for other fungi, indicating that this is not a unique feature of the genus Trichoderma. The presence of melanin-type pigments in spores is supposed to confer resistance to lysis (Bull, 1970). It is therefore not known whether there are differences between Trichoderma and other closely related prey fungi, which would offer an explanation as to how Trichoderma can distinguish between its own cell wall and that of the prey.

\section{Chitinases}

All fungal chitinases described so far are members of GH family 18. While other filamentous fungi usually have between 10 and $20 \mathrm{GH}$ family 18 proteins, T. atroviride has 29 and T. virens has 36. This is the highest number of chitinases described for any filamentous fungus so far. GH 18 chitinases can be categorized into subgroups A, B and C based on the amino acid sequences of their GH 18 modules (Seidl, 2008). Fig. 1 shows a schematic representation of the chitinolytic system of Trichoderma. Based on a comparison to chitinases with known protein structures, the members of the three subgroups have different substratebinding clefts. Subgroup A and C chitinases have rather tunnel-shaped substrate-binding clefts implying exo-activity, whereas subgroup B chitinases have more shallow and open binding clefts mostly found in endo-acting enzymes (Eijsink et al., 2008). In exo-acting enzymes the accessible ends of single polymer chains are threaded through the tight clefts or tunnels while disaccharides are being cleaved off at the catalytic centre. The open clefts of endo-acting enzymes enable them to attach to the polymer surface and cleave the chains in order to supply the exo-acting enzymes with new ends. Further, chitinases from the three subgroups also have different modular structures (Seidl, 2008). Subgroup A chitinases do not contain CBMs, whereas many subgroup B chitinases have CBMs at their C-termini. The CBMs of subgroup B chitinases from Trichoderma spp. belong exclusively to CBM family 1 and have been shown to possess celluloseand chitin-binding properties. Subgroup $\mathrm{C}$ chitinases contain CBM 18 (chitin-binding) and CBM 50 (LysM) modules. LysM modules were first described in bacteria, where they were found to bind to the peptidoglycan of bacterial cell walls. In plants LysM modules were more recently detected in LysM-containing receptor-like kinases (LYKs). They bind to chitinous substrates and were shown to be involved in plant defence responses against fungal pathogens (Knogge \& Scheel, 2006) as well as in symbiotic interactions between the legume plant Medicago truncatula and nitrogen-fixing bacteria (Sinorhizobium meliloti). In fungi LysM modules are predominantly found in subgroup $\mathrm{C}$ chitinases and in proteins that solely consist of a signal peptide and multiple 
Table 1. Expanded GH families in the mycoparasites $T$. atroviride and $T$. virens

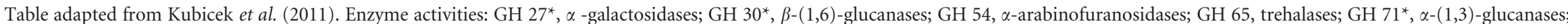

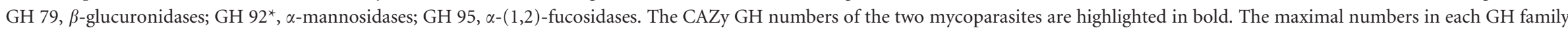

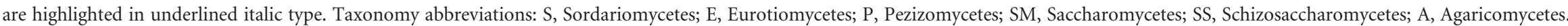

\begin{tabular}{|c|c|c|c|c|c|c|c|c|c|c|c|c|c|c|}
\hline \multirow[t]{2}{*}{ Fungus } & \multirow{2}{*}{ Taxonomy } & \multirow{2}{*}{$\begin{array}{c}\text { Chitinases } \\
\text { GH } 18^{\star}\end{array}$} & \multirow{2}{*}{$\begin{array}{c}\text { Chitosanases } \\
\text { GH } 75^{\star}\end{array}$} & \multicolumn{3}{|c|}{$\beta$ - $(1,3)$-Glucanases } & \multicolumn{8}{|c|}{ Others } \\
\hline & & & & GH $55^{\star}$ & GH $64^{*}$ & GH $81^{\star}$ & GH $27^{\star}$ & GH $30^{*}$ & GH 54 & GH 65 & GH $71^{\star}$ & GH 79 & GH $92^{\star}$ & GH 95 \\
\hline Trichoderma atroviride & $S$ & 29 & $\underline{5}$ & 8 & $\underline{3}$ & 2 & 9 & $\underline{2}$ & $\underline{2}$ & $\underline{2}$ & 4 & 4 & $\underline{8}$ & $\underline{4}$ \\
\hline Trichoderma virens & $S$ & $\underline{36}$ & $\underline{5}$ & $\underline{10}$ & $\underline{3}$ & 1 & $\underline{10}$ & $\underline{2}$ & $\underline{2}$ & $\underline{2}$ & 5 & 4 & $\overline{7}$ & $\underline{4}$ \\
\hline $\begin{array}{l}\text { Trichoderma reesei } \\
\text { PEZIZOMYCOTA }\end{array}$ & S & $\overline{20}$ & 3 & 6 & $\underline{3}$ & 2 & 8 & $\underline{2}$ & $\underline{2}$ & $\underline{2}$ & 4 & 4 & 7 & $\underline{4}$ \\
\hline Aspergillus nidulans & $\mathrm{E}$ & 19 & 2 & 6 & 0 & 1 & 3 & 0 & 0 & 0 & 5 & 4 & 6 & 1 \\
\hline Aspergillus niger & $\mathrm{E}$ & 14 & 2 & 3 & 0 & 1 & 3 & 1 & 1 & 1 & 5 & 1 & 5 & 3 \\
\hline Fusarium graminearum & $\mathrm{S}$ & 19 & 1 & 3 & 2 & 1 & 5 & 0 & 1 & 1 & $\underline{7}$ & 3 & 5 & 2 \\
\hline Magnaporthe grisea & $S$ & 14 & 1 & 3 & 1 & 2 & 1 & 1 & 0 & 0 & $\overline{4}$ & $\underline{10}$ & 2 & 1 \\
\hline Nectria haematococca & $\mathrm{S}$ & 28 & 2 & 5 & 2 & 1 & 4 & 0 & 1 & 0 & 0 & $\overline{1}$ & 6 & 1 \\
\hline Neurospora crassa & $\mathrm{S}$ & 12 & 1 & 6 & 2 & 1 & 2 & 1 & 1 & 0 & 5 & 1 & 0 & 3 \\
\hline Penicillium chrysogenum & $\mathrm{E}$ & 9 & 1 & 3 & 2 & 1 & 0 & 1 & 1 & 0 & 6 & 2 & 2 & 0 \\
\hline Podospora anserina & $S$ & 20 & 1 & 7 & 1 & 1 & 3 & 8 & 0 & 0 & 3 & 5 & 4 & 1 \\
\hline $\begin{array}{l}\text { Tuber melanosporum } \\
\text { Other ASCOMYCETES }\end{array}$ & $\mathrm{P}$ & 5 & 1 & 2 & 0 & $\underline{3}$ & 0 & 0 & 0 & 1 & 0 & 0 & 1 & 0 \\
\hline Saccharomyces cerevisiae & SM & 2 & 0 & 0 & 0 & 2 & 0 & 0 & 0 & 1 & 0 & 0 & 0 & 0 \\
\hline $\begin{array}{l}\text { Schizosaccharomyces pombe } \\
\text { BASIDIOMYCOTA }\end{array}$ & SS & 1 & 0 & 0 & 0 & 1 & 1 & 0 & 0 & 0 & 2 & 0 & 0 & 0 \\
\hline Laccaria bicolor & $\mathrm{A}$ & 10 & 0 & 2 & 0 & 0 & 2 & 8 & 1 & 0 & 0 & 1 & 0 & 2 \\
\hline Phanerochaete chrysosporium & A & 11 & 0 & 2 & 0 & 0 & 1 & 1 & 1 & 1 & 6 & 2 & 4 & 1 \\
\hline Postia placenta & A & 20 & 0 & 6 & 0 & 0 & 2 & 6 & 0 & 0 & 3 & 3 & 2 & 0 \\
\hline
\end{tabular}

${ }^{*}$ Potentially involved in cell wall degradation. 


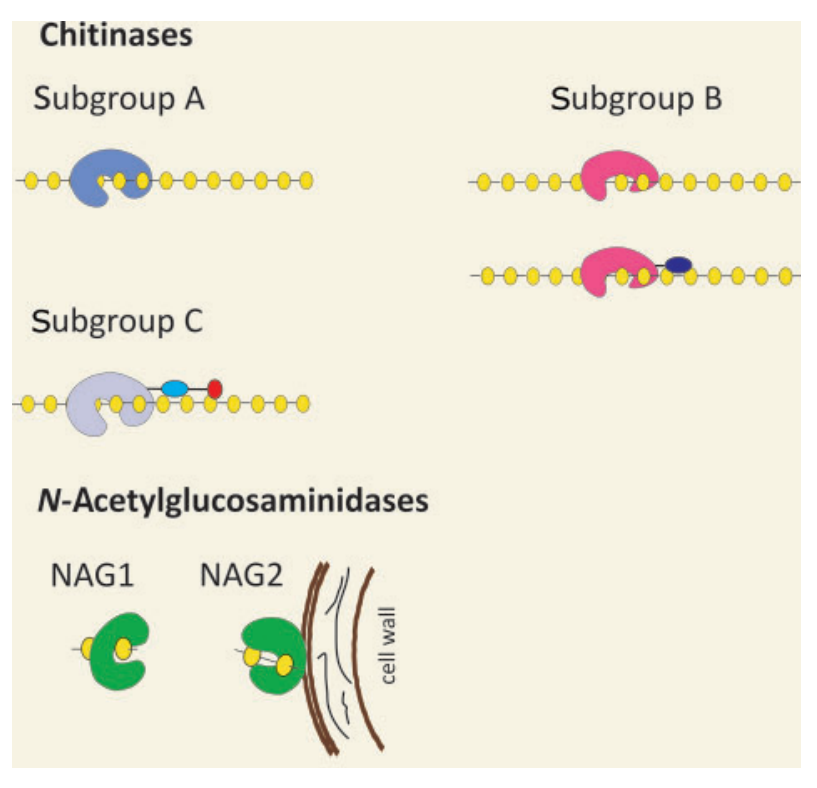

Fig. 1. The chitinolytic enzyme system of Trichoderma. Chitinases belong to $\mathrm{GH}$ family 18 and can be grouped into subgroups $\mathrm{A}, \mathrm{B}$ and $C$. Subgroups $A$ and $C$ are predicted to be exochitinases, which cleave close to the ends of the chitin chain and release $N$ acetylglucosamine dimers or oligomers. Subgroup B chitinases are predicted to be endochitinases and many have a CBM1 domain at their $\mathrm{C}$ terminus. Subgroup $\mathrm{C}$ chitinases contain $\mathrm{CBM}$ 18 and CBM50 domains at the N-terminus of their GH 18 module. Note that subgroup $\mathrm{A}$ and $\mathrm{C}$ (exo)chitinases are predicted to have tighter, more tunnel-shaped substrate-binding clefts than subgroup B (endo)chitinases. It remains to be elucidated which exochitinases in Trichoderma cleave from the reducing and non-reducing ends of the chitin chain, respectively. The smallest degradation product of chitinases is the dimer chitobiose, which is cleaved into monomers by the $N$-acetylglucosaminidases (GH family 20) NAG1 and NAG2. Whereas NAG1 is a secreted into the medium, NAG2 stays attached to the Trichoderma cell wall.

LysMs, but contain no catalytic domain (de Jonge \& Thomma, 2009; Gruber et al., 2011b). In the genomes of Trichoderma spp. the genes encoding LysM proteins are exclusively located next to subgroup C chitinase genes. Moreover, in T. atroviride their expression was found to be largely co-regulated with subgroup $\mathrm{C}$ chitinase genes, suggesting related functions of LysM proteins and subgroup C chitinases (Gruber et al., 2011b).

Elevated numbers of subgroup B and C chitinase genes were found in the genomes of mycoparasitic Trichoderma spp. (Karlsson \& Stenlid, 2008). In general, the presence of CBMs in enzymes enables them to bind more tightly to insoluble substrates (Eijsink et al., 2008). Chitin is an extremely recalcitrant, insoluble substrate and thus binding can be a major limiting factor of enzymic chitin hydrolysis. High numbers of subgroup B and C chitinases suggest an increased potential of these fungi to attack insoluble chitinous substrates.
Despite these indications based on the genome analysis, the most abundantly expressed chitinase in Trichoderma, ECH42 (endochitinase- $42 \mathrm{kDa}$ in T. atroviride; CHIT42 in Trichoderma harzianum; CHT42/Tv-ECH1 in T. virens), interestingly belongs to subgroup A. In a study of chitinases in $T$. reesei a serially numbered nomenclature was used for all 18 detected proteins, and the T. reesei orthologue of T. atroviride ECH42 was named CHI18-5. Phylogenetic analysis showed that $\mathrm{CHI} 18-5$, together with $\mathrm{CHI} 18-7$ (corresponding to $T$. virens $\mathrm{Tv}-\mathrm{ECH} 2$ ), formed a wellsupported clade within subgroup A chitinases. This clade contains most of the characterized chitinases that have been cloned or described from other fungi so far, including Aspergillus nidulans ChiB and Aspergillus fumigatus ChiB1. Thus, ECH42 is not a unique chitinase that is only found in mycoparasites or the genus Trichoderma.

The Trichoderma chitinase CHI36 (CHI18-15 in T. reesei) is different from other fungal chitinases as it was probably acquired in the ancestor of the order Hypocreales by horizontal gene transfer from bacteria (Seidl et al., 2005; Viterbo et al., 2001). It shows low similarity to subgroup B chitinases, but overall it is rather divergent from the other fungal chitinases in this subgroup. Further, there are also one or two proteins/fungal species that belong to family GH 18, but are only distantly related to all chitinases so far described. They were not detected in the study by Seidl et al. (2005) due to the BLAST-search strategy applied and their low similarity to other GH18 proteins. However, during automated annotation of the genomes with the CAZy algorithm, which uses a combination of different algorithms (Cantarel et al., 2009; Henrissat, 1991), two of these proteins were detected in the $T$. reesei genome (CHI18-REL1, protein ID 65162; and CHI18-REL2, protein ID 121355). CHI18-REL1 was recently reported to be an endo- $N$-acetylglucosaminidase (Stals et al., 2010), which is involved in deglycosylation of proteins. These enzymes therefore have potential importance for the functionality of secreted proteins by modifying their glycan moieties. The Neurospora crassa genome also encodes two of these distantly related $\mathrm{GH} 18$ proteins and for one of them the respective gene knockout strain is available only as a heterokaryon from the Neurospora Genome Project (http://www.dartmouth.edu/ neurosporagenome/index. html), which indicates that this could be an essential gene. However, the data of Stals et al. (2010) show that this type of GH18 protein is important for protein deglycosylation, but not for chitin degradation.

\section{Chitosanases}

Chitosanases from GH family 75 are another group of proteins that could be involved in the decomposition of chitinous carbohydrates. Chitosan is the partially deacetylated form of chitin and is found to varying extents in fungal cell walls, where it is probably generated via chitin deacetylases (family CE 4). While most fungi have only one or two G 75 proteins, the mycoparasitic Trichoderma spp. 
have five (Kubicek et al., 2011). However, neither their transcriptional regulation nor their enzymic properties have been studied in mycoparasites so far.

\section{Glucanases}

Besides chitin, the second fibrillar polymer in fungal cell walls is $\beta$-(1,3)-glucan (Latgé, 2007); $\beta$-(1,3)-glucanases have so far been described for GH families 16, 55, 64 and 81. For GH families 55, 64 and 81 an increased number was found in mycoparasitic Trichoderma spp. in comparison to other filamentous fungi (Kubicek et al., 2011). $\beta-(1,3)$ Glucan is $\beta-(1,6)$ branched in the fungal cell wall. $\beta-(1,6)$ Glucanases have so far been described in GH families 5 and 30 , but members of these families can have different activities ranging from mannosidases to cellulases and xylosidases (for details see http://www.cazy.org). The protein TvBGN3, a $\beta$-(1,6)-glucanase from family GH 5 (Djonović et al., 2006), was shown to be important for mycoparasitism of the oomycete Pythium ultimum (which does not contain chitin but solely glucans as the main structural cell wall components). Interestingly, the numbers of GH 5 proteins are not increased in the mycoparasites. Trichoderma spp. have two GH 30 proteins, whereas other ascomycetes have none or one. There are also more members of several other $\mathrm{GH}$ families that are able to act on fungal cell walls, including GH 27 ( $\alpha$-galactosidases), GH $71[\alpha$-(1,3)-glucanases $]$ and GH 92 $(\alpha$-mannosidases) (Kubicek et al., 2011), but none of these has been studied in Trichoderma mycoparasitism yet.

\section{Proteases}

Beside carbohydrate-active enzymes, proteases are also important for the degradation of the fungal cell wall. $T$. atroviride and $T$. virens have more proteases than $T$. reesei. Especially the numbers of subtilisin-like proteases are expanded in the mycoparasites. Different types of proteases, including an aspartyl protease and proteases from the subtilisin-like group like PRB1, were reported to be induced during mycoparasitism before and during contact with the prey in different Trichoderma species (Geremia et al., 1993; Seidl et al., 2009; Suárez et al., 2007). The transcriptomic response of $T$. atroviride to a prey fungus resembles those induced by nitrogen limitation (Seidl et al., 2009). It is, however, unlikely that the fungus has a real nitrogen limitation at this stage. It was therefore proposed that the expression of proteolytic enzymes during the onset of mycoparasitism leads to the formation of nitrogenous metabolites (e.g. peptides) derived from the prey fungus which interact with the nitrogen sensors of T. atroviride. This model is similar to what has been reported for nematophagous fungi, where trapping of the prey was shown to be induced by oligopeptides derived from the nematodes.

Taken together, these data show the diversified capacity to degrade the fungal cell wall as a major trait of mycoparasitic Trichoderma spp. In view of the high numbers of different carbohydrate-modifying enzymes potentially involved in self and non-self fungal cell wall degradation, it is clear that further research will be necessary. In particular more biochemical data about the substrate specificities of carbohydrate-active enzymes will be of great importance. In combination with modern techniques to analyse fungal cell wall composition and structure this will strongly aid in understanding the biological functions of these enzymes and their potential involvement in mycoparasitic attack.

\section{Friend or foe? Regulatory aspects of fungal cell wall degradation by Trichoderma}

\section{Gene expression during mycoparasitism and/or autolysis}

In spite of the large numbers of cell wall degrading enzymes, overexpression of single enzymes under strong, constitutive promoters still leads to increased antifungal activities. This has been demonstrated for the chitinases CHIT33 and CHIT42 (=ECH42) in T. harzianum (Limón et al., 1999, 2004) and CHT42 $(=\mathrm{ECH} 42)$ in T. virens (Baek et al., 1999), as well as for the $\beta$-(1,6)-glucanase TvBGN3 in T. virens (Djonović et al., 2006). Further, addition of cellulose-binding domains (CBM 1) to chitinases enhanced the hydrolysis of insoluble substrates. The respective fungal strains overexpressing the hybrid chitinases also showed increased mycoparasitic activities in comparison to strains overexpressing the native chitinases (Limón et al., 2001, 2004).

Although these data suggest important roles for selected hydrolytic enzymes, evidence arising from gene knockout studies is not so clear-cut, depending on the experimental setup. It should be noted in this context that due to inefficient homologous DNA recombination (HR) in mycoparasitic Trichoderma spp., unfortunately only a few gene knockout studies have been performed. This problem can now be circumvented by creating strains that have defects in the HR machinery, e.g. $\Delta k u 70$ strains, as has already been reported for T. virens (Catalano et al., 2011).

Addition of culture supernatants of mycoparasitic Trichoderma spp. grown on inducing carbon sources (e.g. fungal cell walls) to the growth medium of prey fungi usually results in strong inhibition of their germination and growth. In assays with culture filtrates from strains with knockouts of the chitinase genes ech 42 and cht 42 in $T$. atroviride and $T$. virens, respectively, and the $\beta$ - $(1,6)$ endoglucanase Tvbgn 3 in $T$. virens, a reduction of the growth inhibition could be observed. Correspondingly, in overexpression strains of CHT42 and TvBGN3 an increase of growth inhibition of prey fungi was found (Baek et al., 1999; Djonović et al., 2006; Woo et al., 1999). However, in plate confrontation assays, where the mycelia of Trichoderma and the prey fungus grow towards each other and the two fungi are exposed to each other's lytic enzymes and secondary metabolites, no such obvious role of chitinases could be demonstrated in ech 42 knockout strains of $T$. 
atroviride (Woo et al., 1999), as no differences in comparison to the wild-type were observed in early stages of plate confrontation assays with Rhizoctonia solani and Botrytis cinerea. For Tvgbn 3 knockout strains this type of assay was not reported. The residual chitin content of $R$. solani cell walls was not different between plate confrontation assays with the wild-type and an ech 42 knockout strain of T. atroviride (Carsolio et al., 1999). These data suggest that other chitinases can compensate for the absence of ech42 during mycoparasitism.

Fusion of a gene encoding GFP (green fluorescent protein) to the ech42 promoter yielded a fluorescent signal during mycoparasitism. No expression was observed when a membrane with a $12 \mathrm{kDa}$ cut-off was placed between the two fungi, indicating that diffusible factors trigger chitinase gene expression (Zeilinger et al., 1999). These data clearly showed an involvement of ECH42 in mycoparasitism. However, ech 42 is also induced during growth on chitin and, further, there is strong evidence that ECH42 is involved in autolysis in T. atroviride (Carsolio et al., 1994). Autolysis can be described as an enzymic self-degradation of the cells. It is observed in most filamentous fungi at the late stages of cultures and is affected by ageing, programmed cell death, development, nutrient limitation and numerous other factors (White et al., 2002). Already in the first studies with ech42/ECH42 evidence that this protein is involved in autolysis was detected, but this was not pursued any further. Biomass decline during starvation in ageing shake-flask cultivations was slower in a $T$. atroviride ech 42 knockout strain and faster in overexpressing strains (Carsolio et al., 1999). Also, ech42 expression during carbon starvation was reported (Mach et al., 1999). Taken together, these findings suggest that ECH42 is involved not only in mycoparasitism, which occurs during active growth, but also in autolysis. Surprisingly, the consequences of this duality have not been discussed in the past. In support of this observation, ECH42 is found in the same phylogenetic clade as many proteins that have either been shown to be important for autolysis or whose genes were found to be induced during autolysis and/ or growth on chitin. Proteins that are phylogenetically closely related to ECH42 (see also previous section) include A. nidulans $\mathrm{CHIB}$, which was shown to play an important role in autolysis (Shin et al., 2009; Yamazaki et al., 2007). A. fumigatus CHIB1 is also responsible for the majority of the chitinase activity during the autolytic phase of batch cultures (Jaques et al., 2003). These data suggest that ECH42 and related proteins from the CHI18-5/CHI18-7 clade of subgroup $\mathrm{A}$ are involved in the degradation of chitincontaining carbon sources, ranging from exogenous chitin to prey cell walls during the mycoparasitic attack, but also the mycoparasite's own cell walls during autolysis. ECH42 is clearly not a mycoparasitism-specific chitinase. The fascinating question that arises from these data, namely how ECH42 is targeted to its correct place of action, will have to be answered in future studies.

Similar evidence for a dual involvement in mycoparasitism and the mycoparasite's own cell wall recycling during starvation can be found in the literature for the $\mathrm{N}$ acetylglucosaminidase NAG1. This protein was found to be inducible by mycoparasitic growth conditions (Zeilinger et al., 1999), B. cinerea cell walls, chitin (Mach et al., 1999; Peterbauer et al., 1996) and autolysis (López-Mondéjar et al., 2009). NAG1 belongs to GH family 20 and cleaves predominantly $\mathrm{N}$-acetylglucosamine dimers into monomers. NAG1 is secreted into the medium, but a second GH $20 \mathrm{~N}$-acetylglucosaminidase, NAG2, stays attached to the outside of the cell wall of Trichoderma (López-Mondéjar et al., 2009; Ramot et al., 2004). Single and double knockout strains of nag1 and nag2 in T. atroviride showed no defects in germination, growth, biomass decline during starvation or mycoparasitic potential, but double knockout strains were unable to grow on chitin (López-Mondéjar et al., 2009). These strains were also unable to grow on the dimer chitobiose. The data showed that the $\mathrm{N}$-acetylglucosaminidases NAG1 and NAG2 are essential for the use of chitin as a nutrient source. Despite their different extracellular locations the presence of one of them was sufficient to restore normal growth on chitin. Since the double knockout strains showed no phenotype during growth, autolysis or mycoparasitism, these results showed that the use of chitin as a nutrient source is only of minor importance for these processes. It can be concluded that, similar to ECH42, NAG1 and NAG2 are involved in the decomposition of various kinds of chitinous substrates, but are neither mycoparasitism-specific enzymes, nor determinants for self vs non-self cell wall degradation.

\section{Gene expression of subgroup $\mathbf{C}$ chitinases}

Groups of enzymes with higher numbers in the mycoparasites than in other fungi would be obvious candidates for mycoparasitism-specific enzymes. Subgroup C chitinases are such a group of enzymes. $T$. atroviride has nine subgroup $C$ chitinases and $T$. virens 15 , whereas the saprotroph $T$. reesei has only four. Gene regulation of subgroup $\mathrm{C}$ chitinases in $T$. atroviride and $T$. virens was recently investigated in detail (Gruber et al., 2011a, b). In $T$. atroviride all subgroup $\mathrm{C}$ chitinase genes were induced during mycoparasitism and growth on cell walls of $B$. cinerea but interestingly not during mycoparasitism or by cell walls of $R$. solani. Further, only few of the subgroup C chitinase genes in $T$. atroviride were inducible by chitin, suggesting that other, cell-wall-specific components, e.g. oligosaccharides, might act as inducers of these chitinases. The exact composition of the cell walls of $R$. solani and $B$. cinerea is not known, but it should be noted that ech 42 is induced during mycoparasitism of both prey fungi. Surprisingly, the transcriptional profiles of two subgroup C chitinases, tac 2 and tac6, in T. atroviride pointed to an involvement of these proteins in hyphal growth and network formation, although their modular architectures are similar to the other $T$. atroviride subgroup C chitinases (Gruber et al., 2011b). Only five T. atroviride subgroup C chitinases have orthologues in $T$. virens, indicating high evolutionary rates of these genes. When subjected to the 
same growth conditions as T. atroviride, $T$. virens subgroup C chitinases showed completely different transcription profiles. Complex expression patterns were observed under all tested growth conditions, which were not only dependent on nutritional stimuli, but also on the mode of cultivation and the hyphal zone (central vs peripheral zone of the fungal colony) that was harvested. These data showed that developmental and morphogenetic signals derived from inter- and intra-hyphal interactions also regulate subgroup $\mathrm{C}$ chitinase expression in $T$. virens (Gruber et al., 2011a). It can therefore be concluded that subgroup C chitinases are not mycoparasitism-specific, but are involved in self and non-self cell wall degradation. Different growth morphologies, resulting in altered hyphal interaction and network formation, possibly account for the strong differences in subgroup $\mathrm{C}$ chitinase gene regulation between $T$. atroviride and $T$. virens. Further, these data underline the potential pitfalls of automated genome annotation or generalization of data based on gene numbers. Gene expression data give a first indication of what processes the respective proteins could be involved in, but a detailed biochemical characterization will be necessary to study the substrate specificities of the enzymes and gain further insights into their functions. However, the data obtained for subgroup $\mathrm{C}$ chitinase gene regulation show a further example of chitinases that cannot be generally grouped into those that are regulated by external, nutritional stimuli and those that are regulated by colonyinternal signals. Instead, it seems that these aspects are strongly linked at the regulatory level and therefore probably also at the functional level.

\section{A new paradigm for chitinases in fungal cell wall degradation}

From the data available on fungal chitinases, a scheme is emerging in which most of them are induced during both self and non-self cell wall degradation. This suggests that chitinases cannot be grouped into self- and non-self degrading enzymes. Further, if the same chitinases can participate in self- and non-self cell wall degradation, it remains to be elucidated why the enzymes do not harm healthy Trichoderma hyphae during the mycoparasitic attack. We propose that the accessibility of chitin within the fungal cell wall could be a major determinant. In healthy hyphae, accessibility may be limited by protection conferred by cell wall proteins. The data available so far suggest that Trichoderma chitinases degrade chitin in fungal cell walls irrespective of its origin. This implies that their induction by and action on the substrate may be solely triggered by the accessibility of the substrate. One could envisage the scenario shown schematically in Fig. 2. In this scenario, during mycoparasitism the prey fungus is weakened by a large array of secondary metabolites in combination with hydrolytic enzymes. This aggressive secreted mixture is damaging to the hyphae of other fungi that Trichoderma encounters in its habitat and leads to partial cell death and consequently an increased accessibility

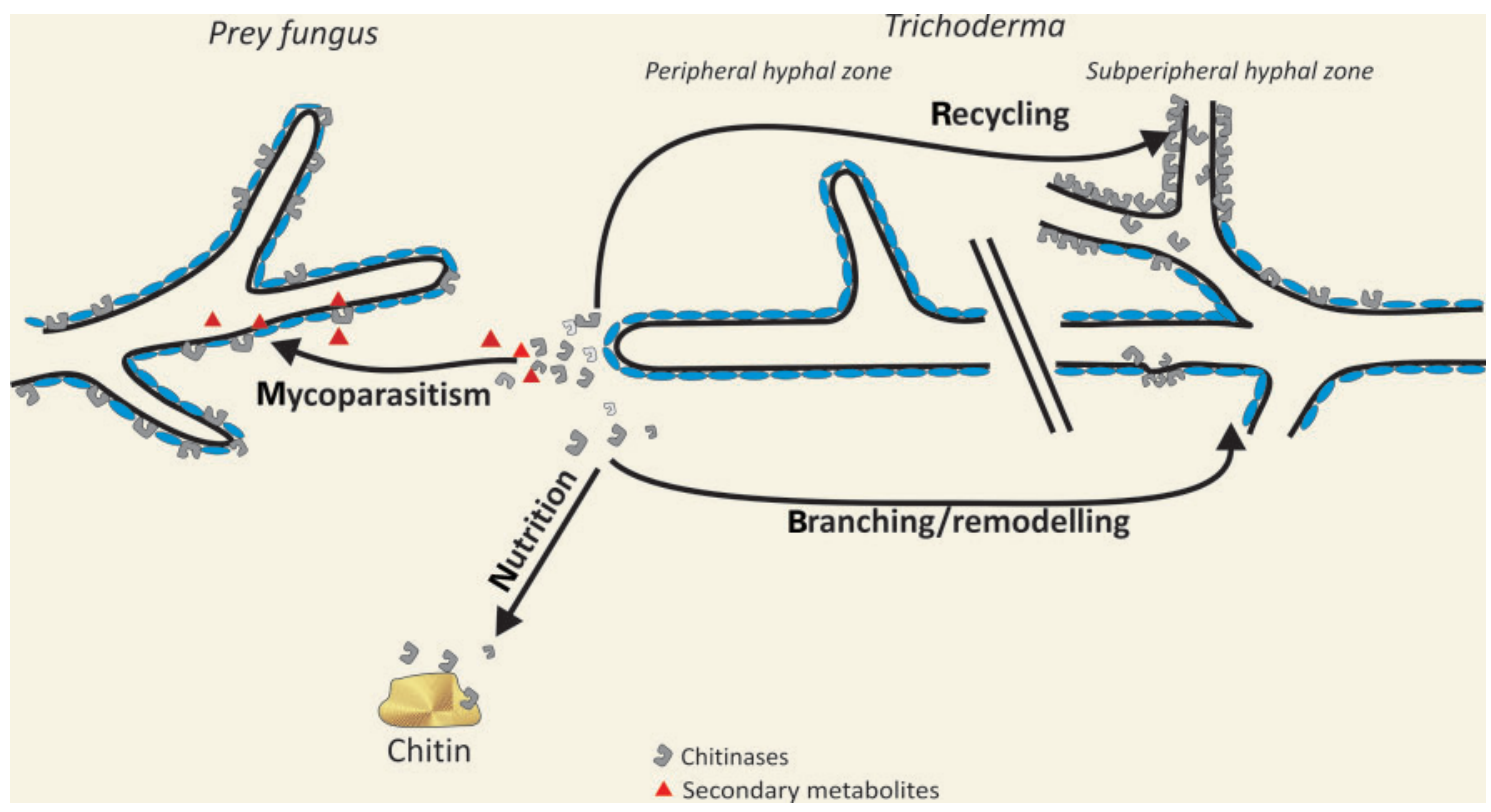

Fig. 2. Model of the roles of chitinases in mycoparasites. Chitinases are secreted into the medium and are involved in different chitinolytic events, depending on the substrate accessibility. The biological functions of chitinases include (i) mycoparasitism (in synergism with secondary metabolites and other enzymes), (ii) exogenous chitin degradation and (iii) remodelling and recycling of the fungus's own cell wall. The accessibility of fungal hyphae is depicted by blue platelets, which exemplify a potential coating of the cell wall polymers with protective substances or other means of cell wall protection. 
of the prey's cell wall carbohydrates. This enables the enzymic release of oligosaccharides, which in turn induce the expression of even more hydrolytic enzymes of Trichoderma and consequently greater damage of the cell wall. Similar enzymes seem to be involved in autolysis of Trichoderma itself. During this process, cell wall polysaccharides are apparently increasingly exposed and consequently degraded. It is also possible that during other processes in fungal colony development, e.g. hyphal branching and fusion, the localized accessibility and de-protection of chitin and other cell wall polysaccharides is a determining factor. From this the question arises of how the fungus differentiates between these growth conditions and how it protects its own cell walls.

Further, the question of how chitinase activity is controlled/suppressed during protein secretion also remains to be addressed in future research. Interestingly, little information is available about how mycoparasitic Trichoderma spp. guard their own cell walls against their hydrolytic enzymes. Hydrophobic cell wall proteins and carbohydrate-binding proteins are two types of protein groups that would be suitable candidates for these protective functions and for which high numbers of genes were found in the genomes of T. atroviride and T. virens (Kubicek et al., 2008, 2011; Seidl-Seiboth et al., 2011). The cell wall protein QID74 from T. harzianum (Rosado et al., 2007) has already been shown to be involved in cell protection in addition to its role in adherence to hydrophobic surfaces, which is reminiscent of the roles of hydrophobins. quid 74 knockout strains were more sensitive to hydrolysis than the wildtype. QID74 is a cysteine-rich protein and consists of multiple repeats of the CXCXC motif (IPR004153). A copy of QID74 is also present in the genomes of the three sequenced species $T$. atroviride, $T$. reesei and $T$. virens but has been found in only very few other fungi; e.g. in the JGI genome database, among approximately 70 sequenced asco- and basidiomycota only Nectria haematococca and Aspergillus aculeatus seem to have versions of this protein (V. Seidl-Seiboth, unpublished results). It should be mentioned that a high number of other small, cysteinerich, secreted proteins were detected in the genomes of $T$. atroviride and T. virens (Kubicek et al., 2011). Their exact functions and their subcellular localization, i.e. whether they adhere to the fungal cell wall, are not known yet. It is possible that these small effector proteins are also involved in aspects of the regulation of cell wall degradation and protection. Carbohydrate-binding proteins could also be involved in these processes, by binding to short oligosaccharides and therefore influencing the induction of cell wall degrading enzymes or even by binding to chitin and therefore masking and protecting it from degradation. In fungal-plant interaction systems such findings have already been reported (de Jonge et al., 2010; van den Burg et al., 2006); it remains to be shown whether similar concepts can be expanded to fungal-fungal systems.

A better understanding of fungal cell wall composition and structure and an integrated knowledge of the enzymes and mechanisms involved in self and non-self degradation will be necessary to understand the roles of carbohydrate-active enzymes during mycoparasitic attack. With the availability of genome sequences and high throughput 'omics' techniques, novel and more comprehensive approaches can now be taken to study mycoparasitism and fungal cell wall degradation in Trichoderma.

\section{Acknowledgements}

Our research is supported by the Austrian Science Fund (grants P20559 and T390 to V.S).

\section{References}

Baek, J. M., Howell, C. R. \& Kenerley, C. M. (1999). The role of an extracellular chitinase from Trichoderma virens Gv29-8 in the biocontrol of Rhizoctonia solani. Curr Genet 35, 41-50.

Benitez, T., Villa, T. G. \& Acha, I. G. (1975). Chemical and structural differences in mycelial and regeneration walls of trichoderma viride. Arch Microbiol 105, 277-282.

Benitez, T., Villa, T. G. \& García Acha, I. (1976). Some chemical and structural features of the conidial wall of Trichoderma viride. Can J Microbiol 22, 318-321.

Benítez, T., Rincón, A. M., Limón, M. C. \& Codón, A. C. (2004). Biocontrol mechanisms of Trichoderma strains. Int Microbiol 7, 249-260.

Bull, A. T. (1970). Inhibition of polysaccharases by melanin: enzyme inhibition in relation to mycolysis. Arch Biochem Biophys 137, 345356.

Cantarel, B. L., Coutinho, P. M., Rancurel, C., Bernard, T., Lombard, V. \& Henrissat, B. (2009). The Carbohydrate-Active EnZymes database (CAZy): an expert resource for glycogenomics. Nucleic Acids Res 37 (Database issue, ), D233-D238.

Carsolio, C., Gutiérrez, A., Jiménez, B., Van Montagu, M. \& HerreraEstrella, A. (1994). Characterization of ech-42, a Trichoderma harzianum endochitinase gene expressed during mycoparasitism. Proc Natl Acad Sci U S A 91, 10903-10907.

Carsolio, C., Benhamou, N., Haran, S., Cortés, C., Gutiérrez, A., Chet, I. \& Herrera-Estrella, A. (1999). Role of the Trichoderma harzianum endochitinase gene, ech42, in mycoparasitism. Appl Environ Microbiol 65, 929-935.

Catalano, V., Vergara, M., Hauzenberger, J. R., Seiboth, B., Sarrocco, S., Vannacci, G., Kubicek, C. P. \& Seidl-Seiboth, V. (2011). Use of a nonhomologous end-joining-deficient strain (delta-ku70) of the biocontrol fungus Trichoderma virens to investigate the function of the laccase gene lcc1 in sclerotia degradation. Curr Genet 57, 13-23.

de Jonge, R. \& Thomma, B. P. (2009). Fungal LysM effectors: extinguishers of host immunity? Trends Microbiol 17, 151-157.

de Jonge, R., van Esse, H. P., Kombrink, A., Shinya, T., Desaki, Y., Bours, R., van der Krol, S., Shibuya, N., Joosten, M. H. \& Thomma, B. P. (2010). Conserved fungal LysM effector Ecp6 prevents chitintriggered immunity in plants. Science 329, 953-955.

Deacon, J. (2006). Fungal Biology. Oxford: Blackwell Publishing.

Djonović, S., Pozo, M. J. \& Kenerley, C. M. (2006). Tvbgn3, a beta-1,6glucanase from the biocontrol fungus Trichoderma virens, is involved in mycoparasitism and control of Pythium ultimum. Appl Environ Microbiol 72, 7661-7670.

Eijsink, V. G., Vaaje-Kolstad, G., Vårum, K. M. \& Horn, S. J. (2008). Towards new enzymes for biofuels: lessons from chitinase research. Trends Biotechnol 26, 228-235. 
Geremia, R. A., Goldman, G. H., Jacobs, D., Ardiles, W., Vila, S. B., Van Montagu, M. \& Herrera-Estrella, A. (1993). Molecular characterization of the proteinase-encoding gene, prbl, related to mycoparasitism by Trichoderma harzianum. Mol Microbiol 8, 603613.

Gruber, S., Kubicek, C. P. \& Seidl-Seiboth, V. (2011a). Differential regulation of orthologous chitinase genes in mycoparasitic trichoderma species. Appl Environ Microbiol 77, 7217-7226.

Gruber, S., Vaaje-Kolstad, G., Matarese, F., López-Mondéjar, R., Kubicek, C. P. \& Seidl-Seiboth, V. (2011b). Analysis of subgroup C of fungal chitinases containing chitin-binding and LysM modules in the mycoparasite Trichoderma atroviride. Glycobiology 21, 122-133.

Henrissat, B. (1991). A classification of glycosyl hydrolases based on amino acid sequence similarities. Biochem J 280, 309-316.

Jaques, A. K., Fukamizo, T., Hall, D., Barton, R. C., Escott, G. M., Parkinson, T., Hitchcock, C. A. \& Adams, D. J. (2003). Disruption of the gene encoding the ChiB1 chitinase of Aspergillus fumigatus and characterization of a recombinant gene product. Microbiology 149, 2931-2939.

Karlsson, M. \& Stenlid, J. (2008). Comparative evolutionary histories of the fungal chitinase gene family reveal non-random size expansions and contractions due to adaptive natural selection. Evol Bioinform Online 4, 47-60.

Knogge, W. \& Scheel, D. (2006). LysM receptors recognize friend and foe. Proc Natl Acad Sci U S A 103, 10829-10830.

Kubicek, C. P., Baker, S., Gamauf, C., Kenerley, C. M. \& Druzhinina, I. S. (2008). Purifying selection and birth-and-death evolution in the class II hydrophobin gene families of the ascomycete Trichoderma/ Hypocrea. BMC Evol Biol 8, 4.

Kubicek, C. P., Herrera-Estrella, A., Seidl-Seiboth, V., Martinez, D. A., Druzhinina, I. S., Thon, M., Zeilinger, S., Casas-Flores, S., Horwitz, B. A. \& other authors (2011). Comparative genome sequence analysis underscores mycoparasitism as the ancestral life style of Trichoderma. Genome Biol 12, R40.

Latgé, J. P. (2007). The cell wall: a carbohydrate armour for the fungal cell. Mol Microbiol 66, 279-290.

Limón, M. C., Pintor-Toro, J. A. \& Benítez, T. (1999). Increased antifungal activity of Trichoderma harzianum transformants that overexpress a 33-kDa chitinase. Phytopathology 89, 254-261.

Limón, M. C., Margolles-Clark, E., Benítez, T. \& Penttilä, M. (2001). Addition of substrate-binding domains increases substrate-binding capacity and specific activity of a chitinase from Trichoderma harzianum. FEMS Microbiol Lett 198, 57-63.

Limón, M. C., Chacón, M. R., Mejías, R., Delgado-Jarana, J., Rincón, A. M., Codón, A. C. \& Benítez, T. (2004). Increased antifungal and chitinase specific activities of Trichoderma harzianum CECT 2413 by addition of a cellulose binding domain. Appl Microbiol Biotechnol 64, 675-685.

López-Mondéjar, R., Catalano, V., Kubicek, C. P. \& Seidl, V. (2009). The beta- $N$-acetylglucosaminidases NAG1 and NAG2 are essential for growth of Trichoderma atroviride on chitin. FEBS J 276, 5137-5148.

Mach, R. L., Peterbauer, C. K., Payer, K., Jaksits, S., Woo, S. L., Zeilinger, S., Kullnig, C. M., Lorito, M. \& Kubicek, C. P. (1999). Expression of two major chitinase genes of Trichoderma atroviride (T. harzianum P1) is triggered by different regulatory signals. Appl Environ Microbiol 65, 1858-1863.

Peterbauer, C. K., Lorito, M., Hayes, C. K., Harman, G. E. \& Kubicek, C. P. (1996). Molecular cloning and expression of the nagl gene
( $N$-acetyl- $\beta$-D-glucosaminidase-encoding gene) from Trichoderma harzianum P1. Curr Genet 30, 325-331.

Ramot, O., Viterbo, A., Friesem, D., Oppenheim, A. \& Chet, I. (2004). Regulation of two homodimer hexosaminidases in the mycoparasitic fungus Trichoderma asperellum by glucosamine. Curr Genet 45, 205213.

Rosado, I. V., Rey, M., Codón, A. C., Govantes, J., Moreno-Mateos, M. A. \& Benítez, T. (2007). QID74 cell wall protein of Trichoderma harzianum is involved in cell protection and adherence to hydrophobic surfaces. Fungal Genet Biol 44, 950-964.

Seidl, V. (2008). Chitinases of filamentous fungi: a large group of diverse proteins with multiple physiological functions. Fungal Biol Rev 22, 36-42.

Seidl, V., Huemer, B., Seiboth, B. \& Kubicek, C. P. (2005). A complete survey of Trichoderma chitinases reveals three distinct subgroups of family 18 chitinases. FEBS J 272, 5923-5939.

Seidl, V., Song, L., Lindquist, E., Gruber, S., Koptchinskiy, A., Zeilinger, S., Schmoll, M., Martínez, P., Sun, J. \& other authors (2009). Transcriptomic response of the mycoparasitic fungus Trichoderma atroviride to the presence of a fungal prey. $B M C$ Genomics 10, 567.

Seidl-Seiboth, V., Gruber, S., Sezerman, U., Schwecke, T., Albayrak, A., Neuhof, T., von Döhren, H., Baker, S. E. \& Kubicek, C. P. (2011). Novel hydrophobins from Trichoderma define a new hydrophobin subclass: protein properties, evolution, regulation and processing. J Mol Evol 72, 339-351.

Shin, K. S., Kwon, N. J., Kim, Y. H., Park, H. S., Kwon, G. S. \& Yu, J. H. (2009). Differential roles of the ChiB chitinase in autolysis and cell death of Aspergillus nidulans. Eukaryot Cell 8, 738-746.

Stals, I., Samyn, B., Sergeant, K., White, T., Hoorelbeke, K., Coorevits, A., Devreese, B., Claeyssens, M. \& Piens, K. (2010). Identification of a gene coding for a deglycosylating enzyme in Hypocrea jecorina. FEMS Microbiol Lett 303, 9-17.

Suárez, M. B., Vizcaíno, J. A., Llobell, A. \& Monte, E. (2007). Characterization of genes encoding novel peptidases in the biocontrol fungus Trichoderma harzianum CECT 2413 using the TrichoEST functional genomics approach. Curr Genet 51, 331-342.

van den Burg, H. A., Harrison, S. J., Joosten, M. H., Vervoort, J. \& de Wit, P. J. (2006). Cladosporium fulvum Avr4 protects fungal cell walls against hydrolysis by plant chitinases accumulating during infection. Mol Plant Microbe Interact 19, 1420-1430.

Viterbo, A., Haran, S., Friesem, D., Ramot, O. \& Chet, I. (2001). Antifungal activity of a novel endochitinase gene (chit36) from Trichoderma harzianum Rifai TM. FEMS Microbiol Lett 200, 169-174.

White, S., McIntyre, M., Berry, D. R. \& McNeil, B. (2002). The autolysis of industrial filamentous fungi. Crit Rev Biotechnol 22, 1-14.

Woo, S. L., Donzelli, B., Scala, F., Mach, R. L., Harman, G. E., Kubicek, C. P., Del Sorbo, G. \& Lorito, M. (1999). Disruption of the ech42 (endochitinase-encoding) gene affects biocontrol activity in Trichoderma harzianum P1. Mol Plant Microbe Interact 12, 419-429.

Yamazaki, H., Yamazaki, D., Takaya, N., Takagi, M., Ohta, A. \& Horiuchi, H. (2007). A chitinase gene, $c h i B$, involved in the autolytic process of Aspergillus nidulans. Curr Genet 51, 89-98.

Zeilinger, S., Galhaup, C., Payer, K., Woo, S. L., Mach, R. L., Fekete, C., Lorito, M. \& Kubicek, C. P. (1999). Chitinase gene expression during mycoparasitic interaction of Trichoderma harzianum with its host. Fungal Genet Biol 26, 131-140. 